\title{
Rare Disorders of Metabolism with Elevated Butyryl- and Isobutyryl-Carnitine Detected by Tandem Mass Spectrometry Newborn Screening
}

\author{
DWIGHT D. KOEBERL, SARAH P. YOUNG, NIELS GREGERSEN, JERRY VOCKLEY, \\ WENDY E. SMITH, DANIEL KELLY BENJAMIN JR, YAN AN, SUSAN D. WEAVIL, \\ SHU H. CHAING, DEEKSHA BALI, MARIE T. McDONALD, PRIYA S. KISHNANI, \\ Y.-T. CHEN, AND DAVID S. MILLINGTON
}

\begin{abstract}
Division of Medical Genetics [D.D.K., S.P.Y., Y.A., D.B., M.T.M., P.S.K., Y.T.C., D.S.M.] and Division of Infectious Disease [D.K.B.], Department of Pediatrics, Duke University Medical Center, Durham, North Carolina 27710, U.S.A.; Research Unit Molecular Medicine, Aarhus University Hospital, 8200 Aarhus N, Denmark [N.G.]; Department of Medical Genetics, Mayo Clinic, Rochester, Minnesota 55905-0001, U.S.A. [J.V.]; Barbara Bush Children's Hospital, Portland, Maine, U.S.A. [W.E.S.]; and North Carolina Newborn Screening Laboratory, Raleigh, North Carolina, U.S.A. [S.D.W., S.H.C.]
\end{abstract}

\begin{abstract}
ABS ing by North Carolina in April 1999. Since then, three infants with short-chain acyl-CoA dehydrogenase (SCAD) and one with isobutyryl-CoA dehydrogenase deficiency were detected on the basis of elevated butyrylcarnitine/isobutyrylcarnitine $\left(\mathrm{C}_{4}{ }^{-}\right.$ carnitine) concentrations in newborn blood spots analyzed by tandem mass spectrometry. For three SCAD-deficient infants, biochemical evaluation included a plasma acylcarnitine profile with markedly elevated $\mathrm{C}_{4}$-carnitine, urine organic acid analysis with markedly elevated ethylmalonic and 2-methylsuccinic acids, and markedly elevated $\left[\mathrm{U}_{-}{ }^{13} \mathrm{C}\right]$ butyrylcarnitine concentrations in medium from fibroblasts incubated with $\left[\mathrm{U}_{-}{ }^{13} \mathrm{C}\right]$ palmitic acid and excess L-carnitine, consistent with classic SCAD deficiency. Two of three infants diagnosed with classic SCAD deficiency remained asymptomatic; however, the third infant presented with seizures and a cerebral infarct at $10 \mathrm{wk}$ of age. All three infants had putatively inactivating mutations in both alleles
\end{abstract}

of the SCAD gene. The highly elevated plasma $\mathrm{C}_{4}$-carnitine levels in the three infants detected by newborn screening tandem mass spectrometry differentiated them from infants and children who were homozygous or compound heterozygous for one of two SCAD gene susceptibility variations; for the latter group the $\mathrm{C}_{4}$-carnitine levels were normal. Isobutyryl-CoA dehydrogenase deficiency in a fourth infant was confirmed after isolated elevation of $\mathrm{C}_{4}$-carnitine in the acylcarnitine profile. (Pediatr Res 54: 219-223, 2003)
EMA, ethylmalonic acid
IBCD, isobutyryl-CoA dehydrogenase
MCAD, medium chain acyl-CoA dehydrogenase
MS/MS, tandem mass spectrometry
SCAD, short chain acyl-CoA dehydrogenase

Newborn screening programs have been expanded with MS/MS to detect up to 20 additional disorders of metabolism compared with previous methods for newborn screening. Disorders of mitochondrial $\beta$-oxidation of fatty acids (especially MCAD deficiency), organic acidemias, and disorders of the urea cycle have been identified before the onset of symptoms

Received August 8, 2002; accepted February 8, 2003.

Correspondence: Dwight D. Koeberl, M.D., Ph.D., Division of Medical Genetics, DUMC 3528, Bell Building Room 237, Trent Drive, Duke University Medical Center, Durham, NC 27710, U.S.A.; e-mail: koebe001@mc.duke.edu

Financial support was provided by National Institutes of Health grant R03 HD42940 (D.K.B.), National Institutes of Health grant K23 RR16060 (P.S.K.), and Genzyme Corporation (S.P.Y.). Financial support for the molecular studies at Research Unit for Molecular Medicine was provided by The Danish Medical Research Council.

DOI: 10.1203/01.PDR.0000074972.36356.89 by newborn screening MS/MS, providing early, beneficial treatment and justifying this approach to newborn screening. However, the evaluation and treatment of some rare disorders of metabolism, including SCAD deficiency, after detection by MS/MS has not been well described.

SCAD deficiency is putatively a very rare disorder, and only 19 cases of this disorder have been described (1-3). Among these cases, two distinct classifications have emerged, either classic SCAD deficiency or variant SCAD. The first group consists of patients with marked ethylmalonic aciduria and methylsuccinic aciduria, markedly elevated $\mathrm{C}_{4}$-carnitine in the acylcarnitine profile, decreased SCAD activity in fibroblasts, and pathogenic mutations in both alleles of the SCAD gene (when reported). Less than half of the 19 reported cases fit 
these criteria, and could be considered classic SCAD deficiency such as the first of two cases reported by Amendt et al. (4). The second, larger group may have intermittent ethylmalonic aciduria, variably decreased SCAD activity, and an SCAD gene susceptibility variation $(625 \mathrm{G} \rightarrow \mathrm{A} / \mathrm{G} \rightarrow \mathrm{A}$ or 511 $\mathrm{C} \rightarrow \mathrm{T} / \mathrm{C} \rightarrow \mathrm{T}$ ) in both alleles, sometimes accompanied by a heterozygous inactivating mutation in one SCAD allele $(2,5$, 6). Biochemical abnormalities may be less pronounced in this second group, and clinical abnormalities show marked heterogeneity $(1-4,7)$. Difficulties with the diagnosis of SCAD deficiency may partially explain the paucity of case reports, especially given that fibroblast analysis may not correspond with muscle SCAD activity (J. Vockley, Mayo Clinic, unpublished data). The overlap of these two presentations for SCAD deficiency has created confusion regarding the diagnosis and outcome of SCAD deficiency.

The presence of elevated $\mathrm{C}_{4}$-carnitines in the acylcarnitine profile can signal either SCAD deficiency or IBCD deficiency in the asymptomatic newborn. The cutoffs used for $\mathrm{C}_{4^{-}}$ acylcarnitine by the newborn screening laboratory might play a role in the ascertainment of patients with variant forms of these disorders.

\section{METHODS}

MS/MS newborn screening. Samples were collected by the Guthrie method on cotton-fiber filter-paper circles and were received and processed by the North Carolina State Laboratory. A one-eighth-inch-diameter disc punched from a single dried blood spot was used for MS/MS processing. Samples were extracted and processed according to established methods $(8,9)$.

Normal newborn screening results were reported to the infant's physician of record at the completion of testing. Abnormal screening results were reported immediately in writing to the physician of record, and further evaluation and follow-up testing were recommended. If the specimens requested for testing were not received by the state laboratory or contact was not made by the physician within $14 \mathrm{~d}$ of notification, the infant's family was notified directly and the recommended testing instructions were relayed. Medical geneticists with expertise in the diagnosis and management of inborn errors of metabolism were available for consultation throughout the screening process.

Biochemical testing and SCAD gene analysis. Urine organic acids were analyzed by capillary gas chromatographymass spectrometry as ethoxime-trimethylsilyl derivatives using a modification of a standard procedure $(10) .{ }^{13} \mathrm{C}_{3}$-EMA (synthesized and characterized in the Duke Mass Spectrometry Facility, data not shown) was added to urine as an internal standard before extraction (approximately $25 \mathrm{mmol} / \mathrm{mol}$ creatinine). Full-scan gas chromatography-mass spectrometry data were acquired over the mass range of 50-600 at a rate of 0.5 s/scan. EMA was quantified by generating ion current chromatograms for m/z 261 (endogenous EMA) and 264 (labeled EMA) from the full-scan data, and comparing the ratio of theses signals to a standard curve (signal ratio versus concentration of added EMA). Plasma acylcarnitines were analyzed either by liquid secondary-ion MS/MS (11-13) with modifications (14) or by electrospray-MS/MS (8).

SCAD activity in muscle biopsy was determined with butyryl-CoA and octanoyl-CoA as substrates, both before and after inactivation with specific antiserum to $\operatorname{MCAD}(4,15)$.

SCAD coding and promoter sequence analysis were performed as described (2). Genomic DNA was prepared from cultured patient fibroblasts by standard methods.

In vitro probe analysis of cultured patient fibroblasts. For in vitro probe analysis confluent skin fibroblasts were incubated with $200 \mu \mathrm{M}\left[\mathrm{U}-{ }^{13} \mathrm{C}\right]$ palmitate (generous gift of Cambridge Isotopes Laboratories, Inc., Andove, MA, U.S.A.) and $400 \mu \mathrm{M}$ L-carnitine in serum-free minimal essential medium containing $0.3 \%(\mathrm{wt} / \mathrm{vol})$ fatty acid-free BSA for the analysis of SCAD deficiency (16). Similarly, skin fibroblasts were incubated with $800 \mu \mathrm{M}\left[\mathrm{U}_{-}{ }^{13} \mathrm{C}\right]$ valine (generous gift of Cambridge Isotopes Laboratories, Inc) or $800 \mu \mathrm{M}$ [3-methyl${ }^{2} \mathrm{H}_{3}$ ]isoleucine (MSD Isotopes Pointe-Claire, Quebec, Canada) and $400 \mu \mathrm{M}$ L-carnitine in serum-free, branch-chain amino acid-free minimal essential medium (Life Technologies) for the analysis of IBCD deficiency. After $120 \mathrm{~h}$ incubation, the culture medium was harvested, and $50 \mu \mathrm{L}$ was mixed with 10 $\mu \mathrm{L}$ of an internal standard containing 45 pmol of acetyl-D, L- $\left[{ }^{2} \mathrm{H}_{9}\right.$-trimethyl $]$ carnitine, $17 \mathrm{pmol}$ of isobutyryl-D, L- $\left[{ }^{2} \mathrm{H}_{9}\right.$ trimethyl]carnitine, 17 pmol of octanoyl-L- $\left[{ }^{2} \mathrm{H}_{3}\right.$-methyl]carnitine, and 17 pmol of palmitoyl- $-\left[-\left[{ }^{2} \mathrm{H}_{3}\right.\right.$-methyl $]$ carnitine. The mixture was spotted onto a one-fourth-inch-diameter cartridge paper disc (no. 900; Schleicher \& Schuell) and left to dry overnight. The discs were extracted with $250 \mu \mathrm{L}$ of methanol, and the extract was dried under $\mathrm{N}_{2}$ and derivatized with 100 $\mu \mathrm{L} 3 \mathrm{~N}$ methanol- $\mathrm{HCl}$ at $50^{\circ} \mathrm{C}$ for $15 \mathrm{~min}$. The methyl ester derivatives were dried under $\mathrm{N}_{2}$, reconstituted in 80:20 methanol: $\mathrm{H}_{2} \mathrm{O}(\mathrm{vol} / \mathrm{vol})$, and analyzed by electrospray-MS/MS using a precursor ion scan of $\mathrm{m} / \mathrm{z} 99$, between $\mathrm{m} / \mathrm{z} 200$ to 500 , at a scan rate of $100 \mathrm{amu} / \mathrm{s}$. A capillary voltage of $3.5 \mathrm{kV}$, a cone voltage of $30 \mathrm{~V}$, collision energy of $22 \mathrm{eV}$, and argon collision gas pressure of $1.5 \times 10^{-3} \mathrm{mBar}$ were used. Concentrations were calculated by peak intensity ratios to the internal standards.

Patients. Newborn screening MS/MS and biochemical testing suggested SCAD deficiency for patients A-C (Table 1), on the basis of markedly elevated $\mathrm{C}_{4}$-carnitine in the blood spot and plasma, and markedly elevated EMA and methylsuccinic acid in urine organic acids. Patient $\mathrm{D}$ also had markedly elevated $\mathrm{C}_{4}$-carnitine in the blood spot and plasma acylcarnitine profiles; however, urine organic acids showed an elevation of isobutyrylglycine rather than EMA and methylsuccinic acids (Table 1).

Statisical analysis. In the analysis of the samples we used generalized estimating equations and assumed an exchangeable correlation coefficient with a Gaussian relationship. We used generalized estimating equations because there were multiple observations for each subject. We repeated our analysis using only the first observation for each subject using a Wilcoxon rank sum test (data not shown), and the results did not differ substantively.

Reported $p$ values are two-tailed, and the analysis was conducted using STATA 6.0 (College Station, TX, U.S.A.). 
Table 1. Biochemical evaluation of patients

\begin{tabular}{ccclcc}
\hline & $\begin{array}{c}\text { Newborn screen: } \\
\mathrm{C}_{4}{ }^{*}(1 \mathrm{st} / 2 \mathrm{nd}) \\
\text { Patient }\end{array}$ & $\begin{array}{c}\text { Follow-up: plasma } \mathrm{C}_{4} \dagger \\
\text { (initial, mean, range, number } \\
\text { of samples) }\end{array}$ & Follow-up: urine organic acids & $\begin{array}{c}\text { Current } \\
\text { age (y) }\end{array}$ \\
\hline $\mathrm{A}$ & $2.5 / 2.8(\mathrm{~d}$ 3/d 32) & $3.9,4.9,3.9-6.0(n=2)$ & Elevated EMA, methylsuccinic & Seizures, hypoglycemia at 3 mo & 3.33 \\
$\mathrm{~B}$ & $2.5 / 2.7(\mathrm{~d} 3 / \mathrm{d} 25)$ & $3.9,4.1,2.6-6.4(n=8)$ & Elevated EMA, methylsuccinic & Normal & 3 \\
$\mathrm{C}$ & $2.3 / 2.3(\mathrm{~d} 3 / \mathrm{d} 23)$ & $2.5,5.2,2.6-7.7(n=7)$ & Elevated EMA, methylsuccinic & Normal & 3.25 \\
$\mathrm{D}$ & $2.3 / 1.5(\mathrm{~d}$ 2/d 20) & $1.5,1.1,0.3-2.8(n=7)$ & Elevated isobutyrylglycine & Normal & 2.92 \\
\hline
\end{tabular}

$* \mathrm{C}_{4}$-carnitine level $(\mu \mathrm{M})$ in the newborn screening blood spot acylcarnitine profile (normal $<1.53$, mean $+4 \mathrm{SD}$ ).

$\dagger \mathrm{C}_{4}$-carnitine level $(\mu \mathrm{M})$ in the initial plasma acylcarnitine profile (normal $<0.46$, mean $+2 \mathrm{SD}$ ).

\section{RESULTS}

Elevated $C_{4-}$ carnitine on newborn screening in SCAD deficiency and in IBCD deficiency. Four infants were evaluated for elevated blood spot $\mathrm{C}_{4}$-carnitine on newborn screening by obtaining a plasma acylcarnitine profile and urine organic acid analysis (Table 1). On follow-up, three of these infants had markedly elevated (at least $3 \times$ the upper limit of normal) EMA and methylsuccinic acid in urine and markedly elevated butyrylcarnitine in plasma consistent with SCAD deficiency (patients A-C). Patient A presented with seizures at $10 \mathrm{wk}$ of age, and a muscle biopsy was performed to measure the muscle SCAD activity, which was deficient $(5.1 \mathrm{mU} / \mathrm{mg}$ with octanoyl-CoA, and unmeasurable with butyryl-CoA). These results indicated SCAD deficiency in patient $\mathrm{A}$, and probable SCAD deficiency in patients $\mathrm{B}$ and $\mathrm{C}$.

As further analysis of SCAD in the above-mentioned patients, sequencing of the SCAD gene was performed (Table 2). Patients A and B were homozygous for a previously described inactivating mutation, $529 \mathrm{~T} \rightarrow \mathrm{C}$, which substitutes arginine for tryptophan at position 153 (5). In spite of this coincidence, these patients are believed to be unrelated on the basis of pedigree information and differences in ethnic background. Patient $\mathrm{C}$ was heterozygous for a nonsense mutation at position 137 , and for a missense mutation that substitutes phenylalanine for serine at position 111. Patients A and B had the normal sequence for the SCAD gene susceptibility variation at nucleotides 511 and 625, and patient $\mathrm{C}$ was heterozygous for the sequence variation at nucleotide 511 (Table 2). In patient $C$, the $511 \mathrm{C} \rightarrow \mathrm{T}$ SCAD gene susceptibility variation was linked with the missense mutation at amino acid position 111.

Patient D was initially evaluated for suspected SCAD deficiency on the basis of elevated blood spot $\mathrm{C}_{4}$-carnitine on newborn screening (Table 1), which suggested increased butyrylcarnitine. However, urine organic acids showed elevated isobutyrylglycine with no elevation of EMA, and this result was consistent with elevated isobutyrylcarnitine and IBCD deficiency. Further confirmation was pursued by in vitro probe analysis of cultured fibroblasts (below).
Differentiation of classic SCAD deficiency from patients with SCAD gene variants. It is possible to ascertain at least some SCAD variants by newborn screening if the cutoff for $\mathrm{C}_{4}$-carnitine is more aggressive than that used in North Carolina (Dr. Steven Levine, Newborn Screening Laboratory, Berkeley, CA, U.S.A., personal communication). Accordingly, we compared the results of repeated plasma $\mathrm{C}_{4}$-carnitine and urinary EMA determinations of the three infants with biochemical profiles typical of classic SCAD deficiency and five patients with variant SCAD. Patients with variant SCAD were homozygotes or compound heterozygotes for SCAD gene susceptibility variations $625 \mathrm{G} \rightarrow \mathrm{A}$ and $511 \mathrm{C} \rightarrow \mathrm{T}$. The plasma $\mathrm{C}_{4}$-carnitine was significantly elevated $(p<0.001)$ by greater than 14-fold in the three infants with classic SCAD deficiency (mean $\pm \mathrm{SD}, 4.63 \pm 1.60 \mu \mathrm{M}, n=17$; mean age $\pm \mathrm{SD}, 332$ \pm 274 d) compared with the variant SCAD patients, for whom plasma $\mathrm{C}_{4}$-carnitine concentrations were within the normal range $(<0.46 \mu \mathrm{M}) . \mathrm{C}_{4}$-carnitine concentrations remained consistently elevated in plasma for the patients with classic SCAD deficiency. The patients with variant SCAD, none of whom were ascertained by newborn screening, were evaluated for various clinical presentations, and initially had mildly elevated EMA and methylsuccinic acid $(<2 \times$ the upper limit of normal) in urine that did not persist on repeated testing. EMA (controls $<16 \mathrm{mmol} / \mathrm{mol}$ creatinine) was always significantly elevated $(p=0.02)$ in classic SCAD-deficient patients (mean $\pm \mathrm{SD}, 160 \pm 130 \mathrm{mmol} / \mathrm{mol}$ creatinine, $n=7$; mean age \pm SD, $603 \pm 188$ d). In summary, patients with classic SCAD deficiency were readily distinguished from those with variant SCAD by the plasma acylcarnitine profile and urine organic acid analysis.

In vitro probe analysis of cultured fibroblasts in SCAD deficiency and in IBCD deficiency. In vitro probe analysis of cultured fibroblasts from the three infants with putative SCAD deficiency demonstrated that $\left[\mathrm{U}^{13}{ }^{13} \mathrm{C}_{4}\right.$-carnitine (absolute concentrations and concentrations normalized to $\left[\mathrm{U}^{13}{ }^{13} \mathrm{C} \mathrm{C}_{16^{-}}\right.$ carnitine) was markedly elevated in the medium compared

Table 2. Sequence analysis of SCAD gene in infants with SCAD deficiency

\begin{tabular}{cllll}
\hline Patient & \multicolumn{1}{c}{$\begin{array}{c}\text { DNA sequence } \\
\text { change }\end{array}$} & Amino acid change & Muscle SCAD & \multicolumn{1}{c}{ SCAD sequence variants } \\
\hline $\mathrm{A}$ & $529 \mathrm{~T} \rightarrow \mathrm{C} / 529 \mathrm{~T} \rightarrow \mathrm{C}$ & W153R* & Deficient $\dagger$ & Normal: 625G/625G and 511C/511C \\
$\mathrm{B}$ & $529 \mathrm{~T} \rightarrow \mathrm{C} / 529 \mathrm{~T} \rightarrow \mathrm{C}$ & W153R* & Not done & Normal: 625G/625G and 511C/511C \\
$\mathrm{C}$ & $332 \mathrm{C} \rightarrow \mathrm{T} / 409 \mathrm{C} \rightarrow \mathrm{T}$ & S111F/Q137X (stop) & Not done & Normal: 625G/625G and 511C $>\mathrm{T} / 511 \mathrm{C}$ \\
\hline
\end{tabular}

* Previously described in Gregersen et al. 5.

$\dagger$ Undetectable activity with butyrylcarnitine substrate with or without immunoactivation with MCAD antiserum; activity with octanoylcarnitine $5.1 \mathrm{mU} / \mathrm{mg}$. 
with controls (Table 3). These results indicated deficient oxidation of butyryl-CoA, consistent with SCAD deficiency.

Cultured fibroblasts from the infant with isobutyrylglycinuria (patient $\mathrm{D}$ in Table 1), analyzed using the in vitro acylcarnitine profiling method with $\left[\mathrm{U}_{-}{ }^{13} \mathrm{C}\right]$ valine as the substrate, demonstrated accumulation of $\left[\mathrm{U}_{-}{ }^{13} \mathrm{C}\right]$ isobutyrylcarnitine in the culture medium (Table 4), consistent with IBCD deficiency. In contrast, $\left[{ }^{2} \mathrm{H}_{3}\right]-\mathrm{C}_{5}$-carnitine did not accumulate in the culture medium when fibroblasts were incubated with [3-methyl- ${ }^{2} \mathrm{H}_{3}$ ]isoleucine (Table 4), indicating that the activity of 2-methylbutyryl-CoA dehydrogenase was normal.

\section{DISCUSSION}

A common dilemma in the evaluation of patients with symptomatic hypoglycemia or those with neurologic symptoms is the interpretation of relatively mild elevations in metabolites in organic acid and acylcarnitine profiles. This difficulty is especially challenging when diagnostic tests require invasive procedures to obtain the relevant tissue for enzyme testing, such as a muscle biopsy in the case of SCAD deficiency. The situation is considerably more complex in the presentation of a normal infant detected by MS/MS newborn screening, such as the elevations of a single metabolite, $\mathrm{C}_{4^{-}}$ carnitine (representing either butyrylcarnitine or isobutyrylcarnitine), found in the patients presented above. Nevertheless, a complete evaluation including analysis of cultured fibroblasts clarified the underlying enzyme deficiency for these three patients, and SCAD deficiency was confirmed by molecular analysis. These patients are characteristic of the classic form of SCAD deficiency, characterized by markedly and persistently elevated $\mathrm{C}_{4}$-carnitine in plasma and EMA and 2-methylsuccinic acids in urine. The diagnosis of three patients with classic SCAD deficiency between the initiation of expanded newborn screening with MS/MS by North Carolina on April 20, 1999, and December 21, 2001, when 320,503 infants were screened (17), suggested an incidence of approximately 1 in 100,000 newborns for SCAD deficiency. Given the detection of only one infant with IBCD deficiency during that interval, this disorder may be quite rare among the population of North Carolina.

We speculate that SCAD deficiency is less rare than previously expected, because the three patients reported here were unrelated and of different ethnicity. The increased detection of rare disorders in newborns using MS/MS can be explained by several possibilities, including random fluctuation in the population or previous underascertainment. Other groups have

Table 3. Diagnosis of SCAD deficiency in cultured skin fibroblasts incubated with $\left[\mathrm{U}^{13}{ }^{3} \mathrm{C}\right]$ palmitate

\begin{tabular}{ccc}
\hline SCAD patient & $\begin{array}{c}{\left[\mathrm{U}^{13} \mathrm{C}\right] \mathrm{C}_{4}} \\
(\mu \mathrm{M})\end{array}$ & {$\left[\mathrm{U}-{ }^{13} \mathrm{C}_{4} /\left[\mathrm{U}-{ }^{13} \mathrm{C}\right] \mathrm{C}_{16}\right.$} \\
\hline A & 1.30 & 26.0 \\
B & 0.45 & 11.3 \\
C & 1.15 & 14.4 \\
Normal controls $(n=22)$ & & $0.52 \pm 0.26$ \\
Mean \pm SD & $0.034 \pm 0.019$ & 1.0 \\
Mean \pm 2 SD & 0.072 & \\
\hline
\end{tabular}

Table 4. Diagnosis of IBCD deficiency in cultured skin fibroblasts incubated with $\left[\mathrm{U}_{-}{ }^{13} \mathrm{C}\right]$ valine and $\left[3-\right.$ methyl $\left.-{ }^{2} \mathrm{H}_{3}\right]$ isoleucine

\begin{tabular}{|c|c|c|}
\hline & {$\left[\mathrm{U}-{ }^{13} \mathrm{C}\right]$-valine probe } & [3-methyl- $\left.{ }^{2} \mathrm{H}_{3}\right]$-isoleucine probe \\
\hline & $\begin{array}{c}{\left[\mathrm{U}_{-}{ }^{13} \mathrm{C}\right] \mathrm{C}_{4} \text {-carnitine }} \\
(\mu \mathrm{M})\end{array}$ & $\begin{array}{c}{\left[{ }^{2} \mathrm{H}_{3}\right]-\mathrm{C}_{5} \text {-carnitine }} \\
(\mu \mathrm{M})\end{array}$ \\
\hline Patient D & 0.93 & 0.13 \\
\hline \multicolumn{3}{|l|}{ Normal controls } \\
\hline Mean \pm SD & $\begin{array}{c}0.013 \pm 0.004 \\
(n=10)\end{array}$ & $\begin{array}{c}0.11 \pm 0.04 \\
(n=5)\end{array}$ \\
\hline Mean +2 SD & 0.022 & 0.18 \\
\hline
\end{tabular}

reported detection of SCAD deficiency by MS/MS newborn screening, although SCAD activity and genetic analysis for those patients were not reported $(18,19)$. However, in Massachusetts four of five infants reportedly had increased urinary ethylmalonate and methylsuccinate or increased plasma $\mathrm{C}_{4^{-}}$ carnitine, which suggested classic SCAD deficiency in approximately 1 in 40,000 births (19). Given the low number of cases in each report and accompanying wide confidence intervals, these two estimates of the incidence of SCAD deficiency are essentially equivalent.

Treatment of SCAD deficiency and IBCD deficiency in the asymptomatic infants presented here is justified on several considerations. Rational treatment, including avoidance of fasting, supplementation of carbohydrates to limit fatty acid or amino acid catabolism when stressed, and supplementation of riboflavin, a cofactor for dehydrogenases $(1,20)$, is available for both disorders. In addition, moderate protein restriction could be beneficial in isobutyryl CoA dehydrogenase deficiency by virtue of reducing the valine intake. A significant risk for disease, including hypoglycemia and CNS damage in SCAD deficiency $(1,2,7)$ and low carnitine with cardiomyopathy in IBCD deficiency (21), has been reported in both disorders. Indeed, patient A presented with seizures as reported in other patients with classic SCAD deficiency. It should be noted, however, that the infarct in patient A might not have been a direct cause of SCAD deficiency.

Each of the SCAD patients reported here has two putatively inactivating mutations in the SCAD gene, either missense or nonsense mutations. Patients A and B were homozygous for $529 \mathrm{~T} \rightarrow \mathrm{C}$, which substitutes arginine for tryptophan at position 153 (5). When the 529T $\rightarrow$ C SCAD variant was expressed in COS-7 cells, the variant protein had very low residual SCAD activity (5). The mutations in patient $\mathrm{C}$ consisted of a nonsense mutation at codon 137, most probably causing rapid degradation of the corresponding mRNA by the nonsense-mediated decay mechanism (22). Alternatively, the protein-if synthesized - will be prematurely terminated with removal of 251 amino acids, including the active center. Likewise, the second mutation in patient $\mathrm{C}$, phenylalanine for serine at position 111, could be predicted to inactivate the SCAD protein because it is a nonconservative amino acid substitution at a position conserved in the mouse and rat SCAD proteins (23). Patient $\mathrm{C}$ also carried the $511 \mathrm{C} \rightarrow \mathrm{T}$ SCAD gene susceptibility variation, previously found to destabilize the SCAD protein (5), and this destabilizing sequence variation was linked with a potentially inactivating mutation, the missense mutation at position 111. Moreover, markedly increased plasma $\mathrm{C}_{4}$-carnitine levels dif- 
ferentiated the infants with classic SCAD deficiency from patients with variant SCAD, either homozygous or compound heterozygous for the common SCAD gene susceptibility variation $(625 \mathrm{G} \rightarrow \mathrm{A}$ and $511 \mathrm{C} \rightarrow \mathrm{T})$. The natural history of patients with SCAD gene susceptibility variation remains uncertain, especially given the variable presentation of patients with these genotypes (2). Ascertainment of variant SCAD by MS/MS newborn screening will depend on the value of the normal cutoff (upper limit of the normal range) used by the newborn screening laboratory, which varies from state to state. However, the $\mathrm{C}_{4}$-carnitine levels in newborn screening may be low enough to preclude detection of those infants with variant $\mathrm{SCAD}$, given the current cutoff value for $\mathrm{C}_{4}$-carnitine in Minnesota (24) and in North Carolina. In North Carolina, we have not detected any infant with variant SCAD by newborn screening using a cutoff value of the mean +4 SDs.

The differentiation between two rare disorders of metabolism in this group of infants with elevated $\mathrm{C}_{4}$-carnitine in newborn screening MS/MS emphasized the need for thorough follow-up evaluations of infants with abnormal blood spot acylcarnitines. Biochemical evaluation consisting of a plasma acylcarnitine profile and urine organic acids, followed by in vitro acylcarnitine profiling and sequencing of the SCAD gene, allowed confirmation of classic SCAD deficiency in three infants. This stepwise approach to the evaluation of SCAD deficiency could obviate the need for a muscle biopsy to demonstrate this disorder, especially in otherwise healthy infants. Finally, a similar approach was applied to establish isobutyrylglycinuria, and quantitative in vitro acylcarnitine profiling provided evidence for a defect of isobutyryl-CoA dehydrogenase in only the second individual shown to have this disorder (21). The ability to recognize and quantify the urinary metabolites for these disorders is dependent on the experience and expertise of the laboratory that performs the follow-up tests after an abnormal newborn screen. We therefore recommend that such tests should be entrusted only to laboratories with proven experience and reliability.

Acknowledgments. The authors thank Dr. Diane Frazier and Dr. Joe Muenzer of the University of North Carolina for their assistance in the follow-up and evaluation of patients with abnormal MS/MS newborn screening in North Carolina. We also thank Steven Hillman and Steven Worthy for their technical assistance.

\section{REFERENCES}

1. Wanders RJ, Vreken P, den Boer MEJ, Wijburg FA, van Gennip AH, Ijlst L 1999 Disorders of mitochondrial fatty acyl-CoA beta-oxidation. J Inherit Metab Dis 22:442-487

2. Corydon MC, Vockley J, Rinaldo P, Rhead WJ, Kjeldsen M, Winter V, Riggs C, Babovic-Vuksanovic D, Smeitink J, De Jong J, Levy H, Sewell AC, Roe C, Matern D, Dasouki M, Gregersen N 2001 Role of common gene variations in the molecular pathogenesis of short-chain acyl-CoA dehydrogenase deficiency. Pediatr Res $49: 18-23$
3. Matern D, Hart P, Murtha AP, Vockley J, Gregersen N, Millington DS, Treem WR 2001 Acute fatty liver of pregnancy associated with short-chain acyl-coenzyme A dehydrogenase deficiency. J Pediatr 138:585-588

4. Amendt BA, Greene C, Sweetman L, Cloherty J, Shih V, Moon A, Teel L, Rhead WJ 1987 Clinical and biochemical studies in two patients. J Clin Invest 79:1303-1309

5. Gregersen N, Winter VS, Corydon MJ, Corydon TJ, Rinaldo P, Ribes A, Martinez G, Bennett MJ, Vianey-Saban C, Bhala A, Hale DE, Lehnert W, Kmoch S, Roig M, Riudor E, Eiberg H, Andresen BS, Bross P, Bolund LA, Kolvraa S 1998 Identification of four new mutations in the short-chain acyl-CoA dehydrogenase (SCAD) gene in two patients: one of the variant alleles, $511 \mathrm{C} \rightarrow \mathrm{T}$, is present at an unexpectedly high frequency in the general population, as was the case for $625 \mathrm{G} \rightarrow \mathrm{A}$, together conferring susceptibility to ethylmalonic aciduria. Hum Mol Genet 7:619-627

6. Corydon MJ, Gregersen N, Lehnert W, Ribes A, Rinaldo P, Kmoch S, Christensen E, Kristensen TJ, Andresen BS, Bross P, Winter V, Martinez G, Neve S, Jensen TG, Bolund L, Kolvraa S 1996 Ethylmalonic aciduria is associated with an amino acid variant of short chain acyl-coenzyme A dehydrogenase. Pediatr Res 39:1059-1066

7. Tein I, Haslam RHA, Rhead WJ, Bennett MJ, Becker LE, Vockley J 1999 Short-chain acyl-CoA dehydrogenase deficiency: a cause of ophthalmoplegia and multicore myopathy. Neurology 52:366-372

8. Rashed MS, Ozand PT, P. Bucknall M, Little D 1995 Diagnosis of inborn errors of metabolism from blood spots by acylcarnitines and amino acid profiling using automated electrospray tandem mass spectrometry. Pediatr Res 38:324-331

9. Rashed MS, Bucknall MP, Little D, Awad A, Jacob M, Alamendi M, Alwattar M, Ozand PT 1997 Screening blood spots for inborn errors of metabolism by electrospray tandem mass spectrometry with microplate batch process and a computer algorithm for automated flagging of abnormal profiles. Clin Chem 43:1129-1141

10. Roe CR, Millington DS, Maltby DA, Bohan TP, Kahler SG, Chalmers RA 1985 Diagnostic and therapeutic implications of medium chain acylcarnitines in the medium chain acyl-CoA dehydrogenase deficiency. Pediatr Res 19:459-466

11. Millington DS, Kodo N, Norwood DL, Roe CR 1990 Tandem mass spectrometry: a new method for acylcarnitine profiling with potential for neonatal screening for inborn errors of metabolism. J Inherit Metab Dis 13:321-324

12. Millington DS, Terada N, Chace DH, Chen Y-T, Ding J-H, Kodo N, Roe CR 1992 The role of tandem mass spectrometry in the diagnosis of fatty acid oxidation disorders. In: Coates PM, Tanaka K (eds) New Developments in Fatty Acid Oxidation. Progress in Clinical and Biological Research, Vol. 375. Wiley-Liss, New York, pp 339-354

13. Millington DS, Chace DH, Hillman SI, Kodo N, Terada N 1994 Diagnosis of Metabolic Disease. In: Matsuo T, Caprioli RM, Gross ML, Seyama Y (eds) Biological Mass Spectrometry: Present and Future. John Wiley and Sons, Ltd, New York, pp $559-579$

14. Van Hove JL, Rutledge SL, Nada MA, Kahler SG, Millington DS 1995 3-Hydroxyisovalerylcarnitine in 3-methylcrotonyl-CoA carboxylase deficiency. J Inherit Metab Dis 18:592-601

15. Coates PM, Hale DE, Finocchiaro G, Tanaka K, Winter SC 1988 Genetic deficiency of short-chain acyl-coenzyme A dehydrogenase in cultured fibroblasts from a patient with muscle carnitine deficiency and severe skeletal muscle weakness. J Clin Invest 81:171-175

16. Ventura FV, Costa CG, Struys EA, Ruiter J, Allers P, Ijlst L, Tavares de Almeida I, Duran M, Jakobs C 1999 Quantitative acylcarnitine profiling in fibroblasts using $\left[\mathrm{U}-{ }^{13} \mathrm{C}\right]$ palmitic acid: an improved tool for the diagnosis of fatty acid oxidation defects. Clin Chim Acta 281:1-17

17. Muenzer J, Frazier DM, McCandless S, Weavil SD, Moore EG, Millington DS, Koeberl DD, Chaing SH 2002 Incidence and false positive rates for metabolic disorders detected by tandem mass spectroscopy newborn screening. 5th Meeting of the International Society for Neonatal Screening, Genova, Italy, 26th -29th June 2002

18. Marsden D, Zytkovicz T, Larson C, Shih V, Grady G 2000 Spectrum of expression of MCAD and SCAD detected by newborn screening. J Inherit Metab Dis 23(suppl 1): 14

19. Zytkovicz TH, Fitzgerald EF, Marsden D, Larson CA, Shih V, Johnson DM, Strauss AW, Comeau AM, Eaton RB, Grady GF 2001 Tandem mass spectrometric analysis for amino, organic, and fatty acid disorders in newborn dried blood spots: a two-year summary from the New England newborn screening program. Clin Chem 47:19451955

20. Gregersen N, Andresen BS, Corydon MJ, Corydon TJ, Olsen RK, Bolund L, Bross P 2001 Mutation analysis in mitochondrial fatty acid oxidation defects: exemplified by acyl-CoA dehydrogenase deficiencies, with special focus on genotype-phenotype relationship. Hum Mutat 18:169-189

21. Roe CR, Cederbaum SD, Roe DS, Mardach R, Galindo A, Sweetman L 1998 Isolated isobutyryl-CoA dehydrogenase deficiency: an unrecognized defect in human valine metabolism. Mol Genet Metab 65:264-267

22. Frischmeyer PA, Dietz HC 1999 Nonsense-mediated mRNA decay in health and disease. Hum Mol Genet 8:1893-1900

23. Kelly CL, Hinsdale ME, Wood PA 1993 Cloning and characterization of the mouse short-chain acyl-CoA dehydrogenation cDNA. Genomics 18:137-140

24. Nagan N, Kruckeberg KE, Tauscher AL, Snow-Bailey K, Rinaldo P, Matern D 2002 The frequency of short-chain acyl-CoA dehydrogenase (SCAD) gene variants in the general population and correlation with the butyrylcarnitine concentration in newborn blood spots. 2002 Society for Inherited Metabolic Disorders Annual Meeting, Asilomar Conference Center, March 3-6 